\title{
Modeling and simulation of the mass flow and reaction kinetics in a continuous Kamyr steam/liquor phase digester
}

\author{
FINN ARE MICHELSEN† and BJARNE A. FOSS $\dagger$
} Keywords: First principles modeling, distributed process, chemical pulping continuous
process, flow dynamics, reaction dynamics

The motion of the mass in a continuous digester can be described by the NavierStokes equations together with mass balances for the chip plug and the free liquor. The component balances for the wood and liquor materials fulfil the mass balances. These equations are solved to help understanding the dynamics connected to motion and chemical reactions taking place in the digester. Simulated profiles are shown in this paper.

\section{Introduction}

The main unit in the cooking system of a pulping mill is the digester. Wood chips from the chip storage are cooked and fed continuously through the reactor for further refinement in the mill before paper making operations.

The quality of the pulp is mainly described by the Kappa number which is a measurement of the residual lignin in the pulp. A typical plant problem is keeping the variations in the Kappa number sufficiently small. As usual in large chemical processes, the measurements of process variables in pulping plants are few and often noisy. Typical control problems include chip level control.

The quality of the pulp coming from the digester is widely accepted as depending mainly on the chip quality, the temperature history of the chips through the vessel, the liquor concentration of active ions, the compaction of the wood chips and the residence time of the chips in the vessel. Especially, the two last quantities are not measured on a continuous basis, and their interactive effects are not totally understood. Both compaction and the residence time of chips are commonly controlled by the chip level, based on the idea that at constant production rate, a constant chip level assures a constant residence time and hence constant compaction. These two very important process variables are dependent variables that have to be distinguished. Understanding and controlling the process in the digester requires knowledge of what is happening in the vessel and how it can be influenced. Such knowledge can be achieved by developing first principles models, such as mass and momentum balances, and simulating them on a computer. The purpose of making a new model is also to make a platform for control structure study. This, then this makes a tool for achieving better pulp quality.

The published literature on modeling pulping digesters mainly includes detailed descriptions of the complex kinetic reactions related to the delignification and degradation of carbohydrates, diffusion and penetration phenomena in the process of

Received 23 June 1993.

$\dagger$ The University of Trondheim, Department of Engineering Cybernetics, The Norwegian Institute of Technology, 7034 Trondheim, Norway.

A preliminary version of this paper was presented at the SIMS '93 Simulation Conference, Kongsberg, June 9-11, 1993. 
transporting liquid into the chips, and early ad hoc models (H-factor models). It was Smith and Williams (1974) who first applied the complex chemistry of pulping in a simple fashion and developed a kinetic model in the form of dynamic mass balances for the main components undergoing reaction. This model was later modified by Christensen et al. (1982). The only reported description of the flow phenomena including the compaction of chips was done by Härkönen (1984) in his doctoral thesis, 'A mathematical model for two-phase flow'. This model is steady state and does not describe the delignification and degradation reactions specifically. It neglects the pressure in the free liquid and ignores some effects which might be interesting to examine, such as inertia effects, dispersion and eddy diffusion. This model also does not have the chip level as a state variable. In studying the operational problems in the digester, we assume the chip level to be of essential importance in a dynamic model.

The proposed model is an integration of the ideas behind the work by Smith and Williams (1974), Christensen et al. (1982) and Härkönen (1984). The physical motion in the digester is described as a case of flow through porous media (Bear 1972). The mass flow is then regarded as a two-phase flow where the incompressible free liquid flows through a compressible moving bed of chips forming a column that can be thought of as an isotropic continuum, i.e a porous medium. In addition to compensating for the deficiencies of the model by Härkönen (1984), the proposed model consists of scaled equations that are formulated in the terms of flow dynamics. Unlike the models referred to above, the proposed model does not include energy balance to describe temperature profiles neither does it describe conic side walls. Furthermore no cross-sectional variations are assumed. The assumptions in the model lie also in the conceptual model which is described in section 2, and in empiric algebraic relations such as the reaction kinetics, mass transfer-, friction- and compressibility coefficients, valve characteristics etc.

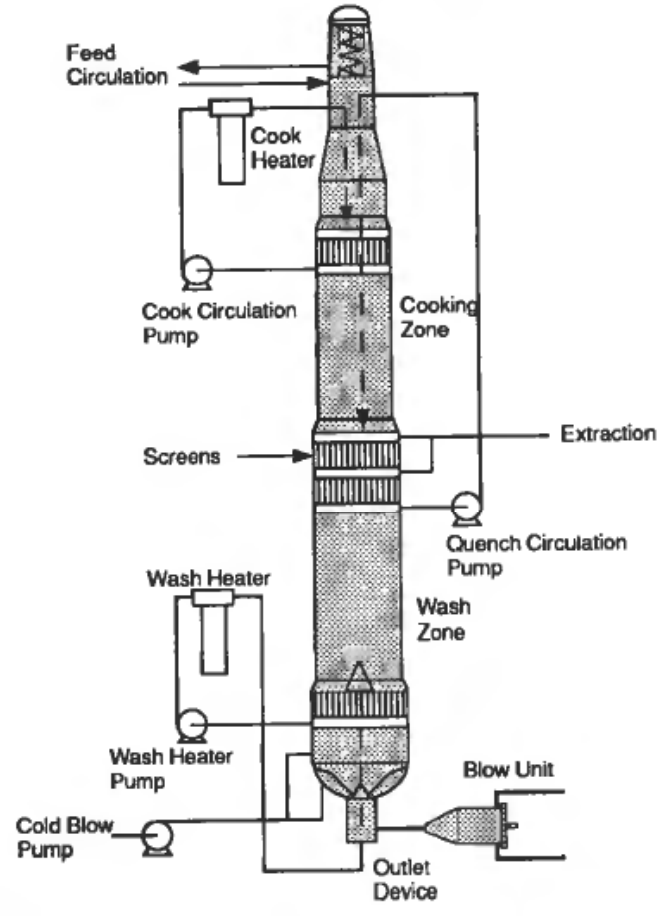

Figure 1. Kamyr steam/liquid phase digester for continuous kraft cooking. 


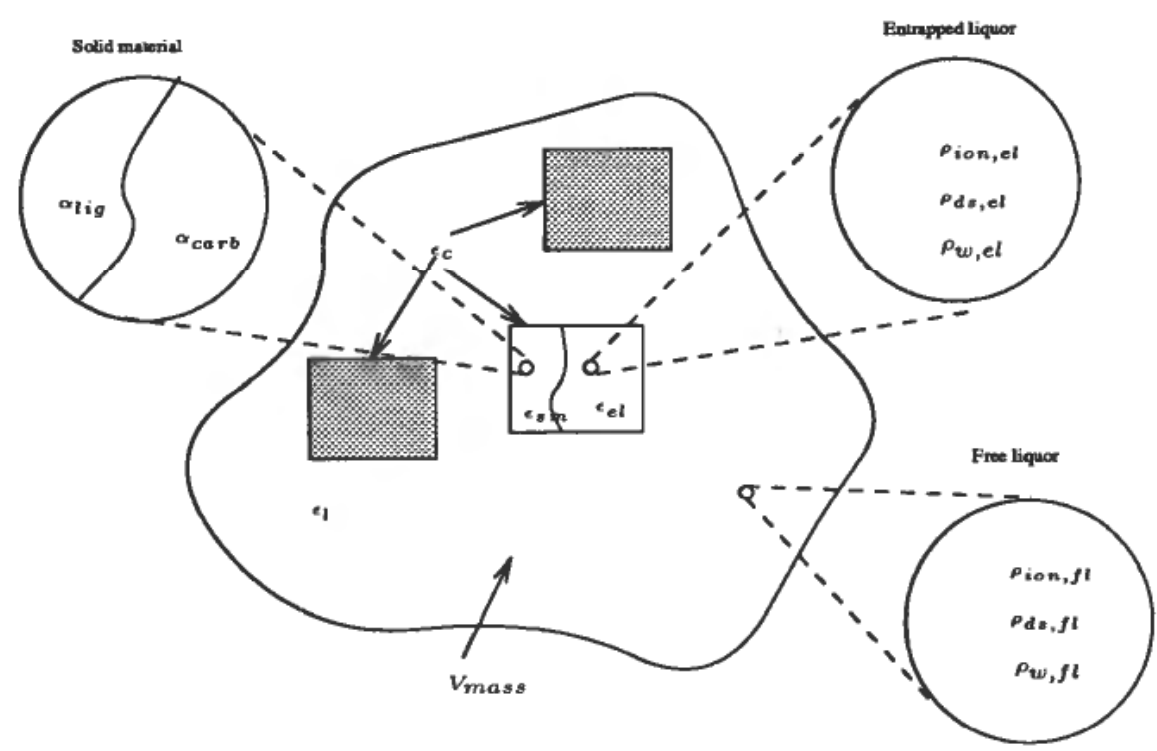

Figure 2. The volume and mass fractions and densities in the model.

The continuation of this paper is as follows. First, fundamental volumetric and mass relationships are established. Second, the proposed model is presented. Third, a short description of the solution method is given. Then, simulation results are shown. The paper ends with a discussion.

\section{Volumetric, mass and density relationships}

Inside the digester (see Fig. 2), chips have volume fraction related to an elementary volume of mass given by

$$
\epsilon_{\mathrm{c}}=V_{\text {chips }} / V_{\text {mass }}
$$

$\epsilon_{\mathrm{c}}$ is thus an expression of the density of wood chips in the mass. This is what can be regarded as the solid matrix of the porous mass media. The interstitial void space between the chips is filled with steam above the liquid level and free liquid, i.e free to circulate, below. This liquid has volume fraction $\epsilon_{1}$ in the mass given by

Hence, we have the relation

$$
\epsilon_{1}=V_{\mathrm{fl}} / V_{\text {mass }}
$$

$$
\epsilon_{\mathrm{c}}+\epsilon_{1}=1 \text {. }
$$

The compaction, $c$, of chips expresses how much the chips are compressed inside the digester compared to in the feed, and can be written as

$$
c=\epsilon_{\mathrm{c}} / \epsilon_{\mathrm{c}, \mathrm{feed}}
$$

A chip has a certain fraction of solid material and entrapped liquid. These are defined by

$$
\begin{aligned}
\epsilon_{\mathrm{sm}} & =V_{\mathrm{sm}} / V_{\mathrm{ODW}} \\
\epsilon_{\mathrm{el}} & =V_{\mathrm{el}} / V_{\mathrm{ODW}}
\end{aligned}
$$

where $V_{\text {ODw }}$ is the known volume of one single oven-dry wood (ODW) chip. This volume and the mass of one single oven-dry chip, $m_{\mathrm{ODw}}$, is, according to Smith and 
Williams (1974), chosen as a reference system for the model. The advantage of this reference system is that for example the content of residual lignin is related to uncooked chips and therefore gives a measurement of how much the chips have been cooked. The density, $\rho_{\mathrm{ODW}}=m_{\mathrm{ODW}} / V_{\mathrm{ODW}}$, of an uncooked chip is then a known parameter in the model. Another interesting fact which fits into this model is that the volume of a single chip is maintained practically unchanged throughout the process in the digester despite the compaction. This is widely accepted and has been shown through laboratory experiments by Härkönen (1984). The consequence of this gives the relation

$$
V_{\text {chips }}=n \cdot V_{\text {ODw }}
$$

where $n$ is the number of chips in an elementary volume of mass. We assume complete penetration of liquid in the chips. Hence

$$
\epsilon_{\mathrm{sm}}+\epsilon_{\mathrm{el}}=1 \text {. }
$$

The solid material of the chips consists basically of lignin and carbohydrates. The high and low reactive lignin is lumped together in one single lignin component and so are the separate carbohydrate species, i.e. the celluloses and the hemicelluloses. The amount of extractives, such as terpenes, in the chips entering the digester is negligible due to the small amount in the unheated chips and to the rapid removal during the further preheating in the preimpregnation vessel which is normally used upstream of a steam/liquor phase digester. To benefit from the structure of the mathematical description of the reaction kinetics in the model by Christensen et al. (1982), mass fractions of lignin and carbohydrates are used. They are defined as

$$
\begin{aligned}
\alpha_{\text {lig }} & =m_{\text {lig }} / m_{\mathrm{ODW}} \\
\alpha_{\mathrm{carb}} & =m_{\mathrm{carb}} / m_{\mathrm{ODW}}
\end{aligned}
$$

where $m_{\text {lig }}$ is the mass of lignin and $m_{\text {carb }}$ is the mass of carbohydrates in one single chip. The solid material can also be fractioned into volume fractions of lignin and carbohydrates related to the chip volume:

so that

$$
\begin{aligned}
\epsilon_{\text {lig }} & =V_{\text {lig }} / V_{\mathrm{ODW}} \\
\epsilon_{\text {carb }} & =V_{\text {carb }} / V_{\text {ODW }}
\end{aligned}
$$

$$
\epsilon_{\mathrm{lig}}+\epsilon_{\mathrm{carb}}=\frac{V_{\mathrm{sm}}}{V_{\mathrm{ODW}}}=\epsilon_{\mathrm{sm}} \cdot
$$

The density of lignin and carbohydrates in the wood chip can then be expressed by

$$
\begin{gathered}
\rho_{\text {lig, chip }}=\frac{m_{\text {lig }}}{V_{\text {ODW }}}=\frac{V_{\text {lig }}}{V_{\text {ODW }}} \frac{m_{\text {lig }}}{V_{\text {lig }}}=\epsilon_{\text {lig }} \rho_{\text {lig }} \\
\rho_{\text {carb, chip }}=\frac{m_{\text {carb }}}{V_{\text {ODW }}}=\frac{V_{\text {carb }}}{V_{\text {ODW }}} \frac{m_{\text {carb }}}{V_{\text {carb }}}=\epsilon_{\text {carb }} \rho_{\text {carb }} .
\end{gathered}
$$

Further, we have

$$
\begin{gathered}
\rho_{\text {lig, chip }}=\frac{m_{\text {lig }}}{V_{\mathrm{ODW}}}=\frac{m_{\mathrm{lig}}}{m_{\mathrm{ODW}}} \frac{m_{\mathrm{ODW}}}{V_{\mathrm{ODW}}}=\alpha_{\mathrm{lig}} \sigma_{\mathrm{ODW}} \\
\rho_{\text {carb, chip }}=\frac{m_{\text {carb }}}{V_{\mathrm{ODW}}}=\frac{m_{\mathrm{carb}}}{m_{\mathrm{ODW}}} \frac{m_{\mathrm{ODW}}}{V_{\mathrm{ODW}}}=\alpha_{\text {carb }} \rho_{\mathrm{ODW}} .
\end{gathered}
$$


These relationships then give by summing

$$
\epsilon_{\mathrm{lig}}+\epsilon_{\mathrm{carb}}=\left(\frac{\alpha_{\mathrm{lig}}}{\rho_{\mathrm{lig}}}+\frac{\alpha_{\mathrm{carb}}}{\rho_{\mathrm{carb}}}\right) \rho_{\mathrm{ODW}}
$$

Eqns. (8) and (13)-(17) then give the following expression:

$$
\epsilon_{\mathrm{el}}=1-\left(\frac{\alpha_{\mathrm{lig}}}{\rho_{\mathrm{lig}}}+\frac{\alpha_{\mathrm{carb}}}{\rho_{\text {carb }}}\right) \rho_{\mathrm{oDw}}
$$

$\rho_{\text {lig }}$ and $\rho_{\text {carb }}$ are the specific weights of lignin and carbohydrates.

The entrapped liquid consists of active ions, dissolved solids and water. In the same way as for lignin and carbohydrates, the separate species of active ions, i.e. hydroxide, hydrosulphide, anthraquinone, polysulphide ions etc. are lumped together in one single component. Again, to benefit from the model by Christensen et al. (1982), density variables for the ions, the dissolved solids and water are used. They are defined as

$$
\begin{gathered}
\rho_{\mathrm{ion}, \mathrm{el}}=\frac{m_{\mathrm{ion}, \mathrm{el}}}{V_{\mathrm{el}}} \\
\rho_{\mathrm{ds}, \mathrm{el}}=\frac{m_{\mathrm{ds}, \mathrm{el}}}{V_{\mathrm{el}}} \\
\rho_{\mathrm{w}, \mathrm{el}}=\frac{m_{\mathrm{w}, \mathrm{el}}}{V_{\mathrm{el}}} .
\end{gathered}
$$

Equivalently, the free liquid is also composed of active ions, dissolved solids and water. Their densities are defined as

$$
\begin{gathered}
\rho_{\mathrm{ion}, \mathrm{fl}}=\frac{m_{\mathrm{ion}, \mathrm{fl}}}{V_{\mathrm{fl}}} \\
\rho_{\mathrm{ds}, \mathrm{fl}}=\frac{m_{\mathrm{ds}, \mathrm{fl}}}{V_{\mathrm{fl}}} \\
\rho_{\mathrm{w}, \mathrm{fl}}=\frac{m_{\mathrm{w}, \mathrm{fl}}}{V_{\mathrm{fl}}} .
\end{gathered}
$$

Note that all the density variables $(20)-(25)$, which are used as state variables in the model, express mass components related to a time varying volume.

Based on these relationships, the concentration (related to a elementary volume of mass) of lignin, carbohydrates, entrapped active ions, dissolved solids and water, free active ions, dissolved solids and water can be derived,

$$
\begin{gathered}
\rho_{\text {lig, mass }}=n \frac{m_{\text {lig }}}{V_{\text {mass }}}=\frac{m_{\text {lig }}}{m_{\mathrm{ODW}}} \frac{m_{\mathrm{ODW}}}{V_{\mathrm{ODW}}} \frac{V_{\mathrm{ODW}}}{V_{\text {mass }}} n=\alpha_{\mathrm{lig}} \rho_{\mathrm{ODW}} \epsilon_{\mathrm{c}} \\
\rho_{\text {carb, mass }}=n \frac{m_{\text {carb }}}{V_{\text {mass }}}=\frac{m_{\mathrm{carb}}}{m_{\mathrm{ODW}}} \frac{m_{\mathrm{ODW}}}{V_{\mathrm{ODW}}} \frac{V_{\mathrm{ODW}}}{V_{\text {mass }}} n=\alpha_{\text {carb }} \rho_{\mathrm{ODW}} \epsilon_{\mathrm{c}} \\
\rho_{\text {ion, el, mass }}=n \frac{m_{\text {ion,el }}}{V_{\text {mass }}}=\frac{m_{\text {ion, el }}}{V_{\text {el }}} \frac{V_{\mathrm{el}}}{V_{\text {ODW }}} \frac{V_{\mathrm{ODW}}}{V_{\text {mass }}} n=\rho_{\text {ion, el }} \epsilon_{\mathrm{el}} \epsilon_{\mathrm{c}}
\end{gathered}
$$




$$
\begin{aligned}
& \rho_{\mathrm{ds}, \mathrm{el}, \text { mass }}=n \frac{m_{\mathrm{ds}, \mathrm{el}}}{V_{\text {mass }}}=\frac{m_{\mathrm{ds}, \mathrm{el}}}{V_{\mathrm{el}}} \frac{V_{\mathrm{el}}}{V_{\mathrm{ODW}}} \frac{V_{\mathrm{ODW}}}{V_{\text {mass }}} n=\rho_{\mathrm{ds}, \mathrm{el}} \epsilon_{\mathrm{el}} \epsilon_{\mathrm{c}} \\
& \rho_{\mathrm{w}, \text { chip, mass }}=n \frac{m_{\mathrm{w}, \text { chip }}}{V_{\text {mass }}}=\frac{m_{\mathrm{w}, \text { chip }}}{V_{\mathrm{ODW}}} \frac{V_{\mathrm{ODW}}}{V_{\text {mass }}} n=\rho_{\mathrm{w}, \text { chip }} \epsilon_{\mathrm{c}} \\
& \rho_{\text {ion, f1, mass }}=\frac{m_{\text {ion, } \mathrm{fl}}}{V_{\text {mass }}}=\frac{m_{\text {ion, } \mathrm{fl}}}{V_{\mathrm{fl}}} \frac{V_{\mathrm{fl}}}{V_{\text {mass }}}=\rho_{\text {ion, } \mathrm{fl}} \epsilon_{1} \\
& \rho_{\mathrm{ds}, \mathrm{fl}, \mathrm{mass}}=\frac{m_{\mathrm{ds}, \mathrm{fl}}}{V_{\mathrm{mass}}}=\frac{m_{\mathrm{ds}, \mathrm{fl}}}{V_{\mathrm{fl}}} \frac{V_{\mathrm{fl}}}{V_{\mathrm{mass}}}=\rho_{\mathrm{ds}, \mathrm{fl}} \epsilon_{1} \\
& \rho_{\mathrm{w}, \mathrm{fl}, \text { mass }}=\frac{m_{\mathrm{w}, \mathrm{f1}}}{V_{\text {mass }}}=\frac{m_{\mathrm{w}, \mathrm{f1}}}{V_{\mathrm{fl}}} \frac{V_{\mathrm{f} 1}}{V_{\text {mass }}}=\rho_{\mathrm{w}, \mathrm{fl}} \epsilon_{1} .
\end{aligned}
$$

Further, in the mass transfer process between the entrapped and the free liquid, the ions and dissolved solids are replaced by equal volume amount of water, to maintain a constant chip volume in our conceptual model of a wood chip.

\section{Mass, component and momentum balances}

Balance equations are derived from the fundamental conservation laws. The equations are based on the Euler formulation, in which the control volume covering an elementary volume of mass is fixed in space and the modeler observes the mass flowing through this volume. This is in contrast to the Lagrange formulation where the elementary volume is not fixed in space, but changes in size and position as it follows the motion in the flow. This method is suitable for particle tracing. It is obvious that the Euler formulation has limitations in giving an exact description of the whole mass in the digester. This is due to the fact that the chip/liquid mass does not have constant value, since the chip and free liquor levels vary with time. This deficiency does not however prevent us solving the problem by this formulation. We attempt to model the whole mass by separating it into three sections. The top section, which is the upper $1-3 \mathrm{~m}$ of the mass, is described in $\S 3.2$. The outlet section, which covers the mass below the bottom scraper, is described in $\S 3.3$. The middle section, which covers the main part of the mass and is described by partial differential equations, is described in $\S 3.1$. Since the flow of mass is mainly vertical, the equations are only solved in this space direction, called $z$.

\subsection{The middle section}

3.1.1. Mass and component balances. The space of solution in the space direction is from just above the bottom scraper in the outlet device and up to $1-3 \mathrm{~m}$ below the normal chip and liquor levels. Based upon the density definitions given in Eqns. (26)(33), the following balance equations including convection, dispersion, reaction and diffusion, can be derived:

$$
\begin{aligned}
\frac{\partial}{\partial t} \rho_{\mathrm{lig}, \text { mass }}= & \frac{\partial}{\partial t}\left(\epsilon_{\mathrm{c}} \alpha_{\mathrm{lig}} \rho_{\mathrm{ODW}}\right)=-\frac{\partial}{\partial z}\left(\epsilon_{\mathrm{c}} \alpha_{\mathrm{lig}} \rho_{\mathrm{ODw}} v_{\mathrm{c}}\right)-r_{\mathrm{lig}} \rho_{\mathrm{ODW}} \epsilon_{\mathrm{c}} \\
& +D_{\mathrm{c}} \frac{\partial^{2}}{\partial z^{2}}\left(\epsilon_{\mathrm{c}} \alpha_{\mathrm{lig}} \rho_{\mathrm{ODW}}\right)
\end{aligned}
$$




$$
\begin{aligned}
& \frac{\partial}{\partial t} \rho_{\text {carb, mass }}=\frac{\partial}{\partial t}\left(\epsilon_{\mathrm{c}} \alpha_{\mathrm{carb}} \rho_{\mathrm{ODW}}\right)=-\frac{\partial}{\partial z}\left(\epsilon_{\mathrm{c}} \alpha_{\mathrm{carb}} \rho_{\mathrm{ODW}} v_{\mathrm{c}}\right)-r_{\text {carb }} \rho_{\mathrm{ODW}} \epsilon_{\mathrm{c}} \\
& +D_{\mathrm{c}} \frac{\partial^{2}}{\partial z^{2}}\left(\epsilon_{\mathrm{c}} \alpha_{\mathrm{carb}} \rho_{\mathrm{ODW}}\right) \\
& \frac{\partial}{\partial t} \rho_{\text {ion, el, mass }}=\frac{\partial}{\partial t}\left(\epsilon_{\mathrm{c}} \epsilon_{\mathrm{el}} \rho_{\text {ion, el }}\right)=-\frac{\partial}{\partial z}\left(\epsilon_{\mathrm{c}} \epsilon_{\mathrm{cl}} \rho_{\text {ion, el }} v_{\mathrm{c}}\right)-\left(b_{\text {lig }} r_{\text {lig }} \rho_{\text {ODW }} \epsilon_{\mathrm{c}}\right. \\
& \left.+b_{\mathrm{carb}} r_{\mathrm{carb}} \rho_{\mathrm{ODW}} \epsilon_{\mathrm{c}}\right)-D_{\text {ion }}\left(\rho_{\text {ion, el }}-\rho_{\text {ion, }, \mathrm{f}}\right)+D_{\mathrm{c}} \frac{\partial^{2}}{\partial z^{2}}\left(\epsilon_{\mathrm{c}} \epsilon_{\mathrm{el}} \rho_{\text {ion, el }}\right) \\
& \frac{\partial}{\partial t} \rho_{\mathrm{ds}, \mathrm{el}, \text { mass }}=-\frac{\partial}{\partial t}\left(\epsilon_{\mathrm{c}} \epsilon_{\mathrm{el}} \rho_{\mathrm{ds}, \mathrm{el}}\right)=-\frac{\partial}{\partial z}\left(\epsilon_{\mathrm{c}} \epsilon_{\mathrm{el}} \rho_{\mathrm{ds}, \mathrm{el}} v_{\mathrm{c}}\right)+\left(1+b_{\mathrm{lig}}\right) r_{\mathrm{lig}} \rho_{\mathrm{ODW}} \epsilon_{\mathrm{c}} \\
& +\left(1+b_{\mathrm{carb}}\right) r_{\mathrm{carb}} \rho_{\mathrm{ODw}} \epsilon_{\mathrm{c}}-D_{\mathrm{ds}}\left(\rho_{\mathrm{ds}, \mathrm{el}}-\rho_{\mathrm{ds}, \mathrm{fl}}\right)+D_{\mathrm{c}} \frac{\partial^{2}}{\partial z^{2}}\left(\epsilon_{\mathrm{c}} \epsilon_{\mathrm{el}} \rho_{\mathrm{ds}, \mathrm{el}}\right) \\
& \frac{\partial}{\partial t} \rho_{\mathrm{w}, \text { chip, mass }}=\frac{\partial}{\partial t}\left(\epsilon_{\mathrm{c}} \rho_{\mathrm{w}, \text { chip }}\right)=-\frac{\partial}{\partial z}\left(\epsilon_{\mathrm{c}} \rho_{\mathrm{w}, \text { chip }} v_{\mathrm{c}}\right) \\
& +D_{\mathrm{w} 1}\left(\rho_{\mathrm{ion}, \mathrm{el}}-\rho_{\mathrm{ion}, \mathrm{fl}}\right)+D_{\mathrm{w} 2}\left(\rho_{\mathrm{ds}, \mathrm{el}}-\rho_{\mathrm{ds}, \mathrm{fl}}\right)+D_{\mathrm{c}} \frac{\partial^{2}}{\partial z^{2}}\left(\epsilon_{\mathrm{c}} \rho_{\mathrm{w}, \mathrm{chip}}\right) \\
& \frac{\partial}{\partial t} \rho_{\text {ion, fl, mass }}=\frac{\partial}{\partial t}\left(\epsilon_{1} \rho_{\text {ion, fl }}\right)=-\frac{\partial}{\partial z}\left(\varepsilon_{1} \rho_{\text {ion, fl }} v_{l}\right)+D_{\text {ion }}\left(\rho_{\text {ion, el }}-\rho_{\text {ion, fl }}\right) \\
& +D_{1} \frac{\partial^{2}}{\partial z^{2}}\left(\epsilon_{1} \rho_{\text {ion, fil }}\right) \\
& \frac{\partial}{\partial t} \rho_{\mathrm{ds}, \mathrm{fl}, \text { mass }}=\frac{\partial}{\partial t}\left(\epsilon_{1} \rho_{\mathrm{ds}, \mathrm{fl}}\right)=-\frac{\partial}{\partial z}\left(\epsilon_{\mathrm{l}} \rho_{\mathrm{ds}, \mathrm{f1}} v_{1}\right)+D_{\mathrm{ds}}\left(\rho_{\mathrm{ds}, \mathrm{el}}-\rho_{\mathrm{ds}, \mathrm{fl}}\right) \\
& +D_{1} \frac{\partial^{2}}{\partial z^{2}}\left(\epsilon_{1} \rho_{\mathrm{ds}, \mathrm{fl}}\right) \\
& \frac{\partial}{\partial t} \rho_{\mathrm{w}, \mathrm{fl}, \text { mass }}=\frac{\partial}{\partial t}\left(\epsilon_{1} \rho_{\mathrm{w}, \mathrm{fl}}\right)=-\frac{\partial}{\partial z}\left(\epsilon_{1} \rho_{\mathrm{w}, \mathrm{f1}} v_{1}\right) \\
& -D_{\mathrm{w} 1}\left(\rho_{\text {ion, el }}-\rho_{\text {ion, f1 }}\right)-D_{\mathrm{w} 2}\left(\rho_{\mathrm{el}}-\rho_{\mathrm{ds}, \mathrm{fl}}\right)+D_{1} \frac{\partial^{2}}{\partial z^{2}}\left(\epsilon_{1} \rho_{\mathrm{w}, \mathrm{fl}}\right)
\end{aligned}
$$

The sum of Eqns. (34)-(38) is the mass balance of the chips:

$$
\frac{\partial}{\partial t} \rho_{\text {chips }}=-\frac{\partial}{\partial z}\left(\rho_{\mathrm{c}} \epsilon_{\mathrm{c}} v_{\mathrm{c}}\right)-\Delta(D \rho)+D_{\mathrm{c}} \frac{\partial^{2}}{\partial z^{2}}\left(\rho_{\mathrm{c}} \epsilon_{\mathrm{c}}\right)
$$

and the sum of Eqns. (39)-(41) is the mass balance of the free liquid:

$$
\frac{\partial}{\partial t} \rho_{\mathrm{f} 1}=-\frac{\partial}{\partial z}\left(\rho_{1} \epsilon_{1} v_{1}\right)+\Delta(D \rho)+D_{1} \frac{\partial^{2}}{\partial z^{2}}\left(\rho_{1} \epsilon_{1}\right)
$$

where $\Delta(D \rho)=\left(D_{\text {ion }}-D_{\mathrm{w} 1}\right)\left(\rho_{\text {ion, el }}-\rho_{\text {ion, fl }}\right)+\left(D_{\mathrm{ds}}-D_{\mathrm{w} 2}\right)\left(\rho_{\mathrm{ds}, \mathrm{el}}-\rho_{\mathrm{ds}, \mathrm{fl}}\right)$ expresses the net mass transfer between the two phases. 
In addition to the previous parameter and variable definitions, the (rest) of the remainder symbols used are:

$$
\begin{aligned}
r_{\text {lig }}=k_{1} \rho_{\text {ion, el }} \alpha_{\text {lig }} & \begin{array}{l}
\text { chemical reaction coefficients, where } k_{i}, i=1,2 \text { are } \\
\text { the Arrhenius coefficients and } \alpha_{\text {carb }}^{0} \text { is the unreactive }
\end{array} \\
r_{\text {carb }}=k_{2} \rho_{\text {ion, el }}\left(\alpha_{\text {carb }}-\alpha_{\text {carb }}^{0}\right) & \begin{array}{l}
\text { part of the carbohydrates (typically } 30 \%) \text { which is } \\
\text { formed during the stabilization reactions. }[\mathrm{kg} /(\mathrm{kg}
\end{array} \\
& \text { ODW s)] }
\end{aligned}
$$

$b_{\text {lig, }}, b_{\text {carb }}$ stoichiometric coefficients $[\mathrm{kg} / \mathrm{kg}]$ describing the fraction of mass transfer of reaction by the active entrapped ions, i.e the consumption of active ions.

$D_{\mathrm{c}}, D_{1}$ dispersion coefficients $\left[\mathrm{m}^{2} / \mathrm{s}\right]$ which give rise to nonflat velocity profiles in contradiction to the pore plug flow. This results from molecular and turbulene diffusion caused by both axial and radial mixing (Fogler, 1982). Typically $D_{1} \gg D_{c}$.

$D_{\text {ion, }} D_{\mathrm{ds}}$ mass transfer coefficients [ $\left.\mathrm{s}^{-1}\right]$ describing the mass transfer of ions and dissolved solids between the entrapped and the free liquor because of density gradients. According to the references, these are equal. Note that the density variables are defined such that mass transfer occurs whenever $\rho_{\mathrm{i}, \mathrm{el}} \neq \rho_{\mathrm{i}, \mathrm{fl} \text {, }}$ $i=$ ion, ds, even though they do not have the same dimension.

$D_{\mathrm{w} 1}, D_{\mathrm{w} 2}$ mass transfer coefficients [ $\left.\mathrm{s}^{-1}\right]$ describing the interphase mass transfer of water because of density gradients of ions and dissolved solids. Typical are $D_{\mathrm{w} 1} \neq D_{\mathrm{ion}}$ and $D_{\mathrm{w} 2} \neq D_{\mathrm{ds}}$, depending on the specific weight of water compared to the specific weights of the ions and the dissolved solids.

$v_{\mathrm{c}}, v_{1} \quad$ velocity of chips and free liquor $[\mathrm{m} / \mathrm{s}]$

These equations indicate that the mass flow is regarded as a two-phase flow with the chip plug and the free liquor as the two phases.

Note that Eqns. (34)-(41) form a set of eight equations with nine unknowns (densities). As already mentioned, the chip volume is kept constant throughout the whole process in the digester. The process of delignification and degradation of the carbohydrates does, however, influence the compaction, i.e. the volume fraction of chips. This phenomenon is connected to the fact that the chips become softer during cooking due to increased porosity inside every chip. Then they change shape rather than volume. This is described by reduced chip pressure (see later) and is therefore connected to momentum transfer rather than to mass transfer. Thus, the dynamics of $\epsilon_{\mathrm{c}}$ and $\epsilon_{1}$ are independent of the dynamics of the other density variables in Eqns. (34)-(41). They can therefore be separated. 
This separation of the equations gives the following parabolic partial differential equations for the volume fractions of chips and free liquor:

$$
\begin{aligned}
& \frac{\partial \epsilon_{\mathrm{c}}}{\partial t}=-\frac{\partial}{\partial z}\left(\epsilon_{\mathrm{c}} v_{\mathrm{c}}\right)+D_{\mathrm{c}} \frac{\partial^{2} \epsilon_{\mathrm{c}}}{\partial z^{2}} \\
& \frac{\partial \epsilon_{1}}{\partial t}=-\frac{\partial}{\partial z}\left(\epsilon_{1} v_{1}\right)+D_{1} \frac{\partial^{2} \epsilon_{1}}{\partial z^{2}} .
\end{aligned}
$$

By using Eqn. (3), we get the two-phase form of the continuity equation including dispersion:

$$
\frac{\partial v_{1}}{\partial z}=\frac{1}{1-\epsilon_{\mathrm{c}}}\left(\left(v_{1}-v_{\mathrm{c}}\right) \frac{\partial \epsilon_{\mathrm{c}}}{\partial z}+\left(D_{\mathrm{c}}-D_{1}\right) \frac{\partial^{2} \epsilon_{\mathrm{c}}}{\partial z^{2}}\right)
$$

which is elliptic and is used to solve the free liquid velocity.

The reaction kinetics then only influence the mass balances through mass transfer inside the chips. This is expressed by the component balances which are given by:

$$
\begin{aligned}
& \frac{\partial \alpha_{\text {lig }}}{\partial t}=-\frac{\partial}{\partial z}\left(\alpha_{\text {lig }} v_{\mathrm{c}}\right)-r_{\text {lig }}+D_{\mathrm{c}} \frac{\partial^{2} \alpha_{\text {lig }}}{\partial z^{2}} \\
& \frac{\partial \alpha_{\text {carb }}}{\partial t}=-\frac{\partial}{\partial z}\left(\alpha_{\text {carb }} v_{\mathrm{c}}\right)-r_{\text {carb }}+D_{\mathrm{c}} \frac{\partial^{2} \alpha_{\text {carb }}}{\partial z^{2}} \\
& \frac{\partial \rho_{\text {ion, el }}}{\partial t}=-\frac{\rho_{\text {ion, el }}}{\epsilon_{\text {el }}} \frac{\partial v_{\mathrm{c}}}{\partial z}-v_{\mathrm{c}} \frac{\partial \rho_{\text {ion, el }}}{\partial z}-\frac{\rho_{\text {ODw }}}{\epsilon_{\text {el }}}\left(b_{\text {lig }}+\frac{\rho_{\text {ion }, \mathrm{el}}}{\rho_{\text {lig }}}\right) r_{\text {lig }} \\
& \left.+\left(b_{\text {carb }}+\frac{\rho_{\text {ion, el }}}{\rho_{\text {carb }}}\right) r_{\text {carb }}\right)-\frac{D_{\text {ion }}}{\epsilon_{\mathrm{c}} \epsilon_{\text {el }}}\left(\rho_{\text {ion, el }}-\rho_{\text {ion }, \mathrm{fl}}\right)+D_{\mathrm{c}} \frac{\partial^{2} \rho_{\text {ion, el }}}{\partial z^{2}} \\
& \frac{\partial \rho_{\mathrm{ds}, \mathrm{el}}}{\partial t}=-\frac{\rho_{\mathrm{ds}, \mathrm{el}}}{\epsilon_{\mathrm{el}}} \frac{\partial v_{\mathrm{c}}}{\partial z}-v_{\mathrm{c}} \frac{\partial \rho_{\mathrm{ds}, \mathrm{el}}}{\partial z}-\frac{\rho_{\mathrm{ODW}}}{\epsilon_{\mathrm{el}}}\left(\left(\frac{\rho_{\mathrm{ds}, \mathrm{el}}}{\rho_{\mathrm{lig}}}-\left(1+b_{\mathrm{lig}}\right)\right) r_{\mathrm{lig}}\right. \\
& \left.+\left(\frac{\rho_{\mathrm{ds}, \mathrm{el}}}{\rho_{\mathrm{carb}}}-\left(1+b_{\mathrm{carb}}\right)\right) r_{\mathrm{carb}}\right)-\frac{D_{\mathrm{ds}}}{\epsilon_{\mathrm{c}} \epsilon_{\mathrm{el}}}\left(\rho_{\mathrm{ds}, \mathrm{el}}-\rho_{\mathrm{ds}, \mathrm{fl}}\right)+D_{\mathrm{c}} \frac{\partial^{2} \rho_{\mathrm{ds}, \mathrm{el}}}{\partial z^{2}} \\
& \frac{\partial \rho_{\mathrm{w}, \mathrm{chip}}}{\partial t}=-\frac{\partial}{\partial z}\left(\rho_{\mathrm{w}, \mathrm{chip}} v_{\mathrm{c}}\right)+\frac{D_{\mathrm{w} 1}}{\epsilon_{\mathrm{c}}}\left(\rho_{\text {ion, el }}-\rho_{\text {ion,fl }}\right)+\frac{D_{\mathrm{w} 2}}{\epsilon_{\mathrm{c}}}\left(\rho_{\mathrm{ds}, \mathrm{el}}-\rho_{\mathrm{ds}, \mathrm{fl}}\right)+D_{\mathrm{c}} \frac{\partial^{2} \rho_{\mathrm{w}, \mathrm{chip}}}{\partial z^{2}} \\
& \frac{\partial \rho_{\text {ion, fl }}}{\partial t}=-\frac{\partial}{\partial z}\left(\rho_{\text {ion }, \mathrm{fl}} v_{1}\right)+\frac{D_{\text {ion }}}{\epsilon_{1}}\left(\rho_{\text {ion, el }}-\rho_{\text {ion }, \mathrm{fl}}\right)+D_{1} \frac{\partial^{2} \rho_{\text {ion, fl }}}{\partial z^{2}} \\
& \frac{\partial \rho_{\mathrm{ds}, \mathrm{fl}}}{\partial t}=-\frac{\partial}{\partial z}\left(\rho_{\mathrm{ds}, \mathrm{fl}} v_{\mathrm{l}}\right)+\frac{D_{\mathrm{ds}}}{\epsilon^{1}}\left(\rho_{\mathrm{ds}, \mathrm{el}}-\rho_{\mathrm{ds} . \mathrm{fl}}\right)+D_{1} \frac{\partial^{2} \rho_{\mathrm{ds}, \mathrm{fl}}}{\partial z^{2}} \\
& \frac{\partial \rho_{\mathrm{w}, \mathrm{fl}}}{\partial t}=-\frac{\partial}{\partial z}\left(\rho_{\mathrm{w}, \mathrm{fl}} v_{\mathrm{l}}\right)-\frac{D_{\mathrm{w} 1}}{\epsilon_{1}}\left(\rho_{\mathrm{ion}, \mathrm{el}}-\rho_{\mathrm{ion}, \mathrm{fl}}\right)-\frac{D_{\mathrm{w} 2}}{\epsilon_{\mathrm{l}}}\left(\rho_{\mathrm{ds}, \mathrm{el}}-\rho_{\mathrm{ds}, \mathrm{ff}}\right)+D_{1} \frac{\partial^{2} \rho_{\mathrm{w}, \mathrm{fl}}}{\partial z^{2}} \text {. }
\end{aligned}
$$

Note that we have chosen $\rho_{\text {w. chip }}$ as a basic variable (state variable). This is not included in the models of Smith and Williams (1974) and Christensen et al. (1982) and therefore we do not have reliable mass transfer coefficients connected to this density variable. 
The mentioned chip pressure or bed pressure, $p_{\mathrm{c}}[\mathrm{Pa}]$, is defined by the average of the contact forces acting on the points of contact between the chips over a reference surface, divided by this area. This is solved from the equation of state (i.e. the compressibility equation):

$$
p_{\mathrm{c}}=10^{4}\left(\frac{0.356-\epsilon_{\mathrm{c}}}{-0.831+0.139 \ln (\kappa)}\right)^{1.695}
$$

as suggested by Härkönen (1984) from his regression analysis of experimental laboratory data. Note that this equation is valid only for $\epsilon_{\mathrm{c}} \in[0 \cdot 356,1 \cdot 0]$. It relates the compaction to the chip pressure at different degrees of cooking. The last characteristic is expressed by the $\kappa$ number, giving the residual lignin in the mass and related to the foregoing definitions, defined by (Rydholm, 1967):

$$
\kappa=\left(\%\left(\alpha_{\text {lig }} /\left(\alpha_{\text {lig }}+\alpha_{\text {carb }}\right)\right)\right) / 0 \cdot 13 .
$$

In this way, the delignification influences the compaction via the momentum balance, which is derived in the next subsection.

The other pulp quality variable commonly used is the yield, $Y$, which can be expressed by:

$$
Y=\%\left(\alpha_{\text {lig }}+\alpha_{\text {carb }}\right)
$$

3.1.2. Momentum balances. The momentum balances for the phases of vertical flow can be written as the following parabolic partial differential equations when the dispersion terms in the mass balances are omitted:

$$
\begin{aligned}
& \frac{\partial v_{\mathrm{c}}}{\partial t}=-v_{\mathrm{c}} \frac{\partial v_{\mathrm{c}}}{\partial z}-\frac{1}{\rho_{\mathrm{c}} \epsilon_{\mathrm{c}}} \frac{\partial p_{\mathrm{c}}}{\partial z}-\frac{1}{\rho_{\mathrm{c}}} \frac{\partial p_{1}}{\partial z}+g-\frac{1}{\rho_{\mathrm{c}} \epsilon_{\mathrm{c}}} F_{\mu}-\frac{1}{\rho_{\mathrm{c}} \epsilon_{\mathrm{c}}} \Omega\left(v_{\mathrm{c}}-v_{1}\right)+\frac{\eta_{\mathrm{c}}}{\rho_{\mathrm{c}}} \frac{\partial^{2} v_{\mathrm{c}}}{\partial z^{2}} \\
& \frac{\partial v_{1}}{\partial t}=-v_{1} \frac{\partial v_{1}}{\partial z}-\frac{1}{\rho_{1}} \frac{\partial p_{1}}{\partial z}+g-\frac{1}{\rho_{\mathrm{l}} \epsilon_{1}} \Omega\left(v_{1}-v_{\mathrm{c}}\right)+\frac{\eta_{1}}{\rho_{1}} \frac{\partial^{2} v_{1}}{\partial z^{2}} .
\end{aligned}
$$

In addition to the previous description $\rho_{\mathrm{c}}=\left(\alpha_{\text {lig }}+\alpha_{\text {carb }}\right) \rho_{\text {ODw }}+\left(\rho_{\text {ion, ei }}\right) \epsilon_{\text {el }}+\rho_{\mathrm{w}, \text { chip }}$ is the density of the wood chips $\left[\mathrm{kg} / \mathrm{m}^{3}\right], \rho_{1}=\rho_{\mathrm{ion}, \mathrm{fl}}+\rho_{\mathrm{ds}, \mathrm{fl}}+\rho_{\mathrm{w}, \mathrm{fl}}$ is the density of free liquid $\left[\mathrm{kg} / \mathrm{m}^{3}\right], p_{1}$ is the pressure in the free liquid [Pa], $g$ is the constant of gravity $\left[\mathrm{m} / \mathrm{s}^{2}\right]$, $F_{\mu}=4 \mu p_{c} / d$ is dry force of friction between the chip plug and the side walls in the digester [N], where $\mu$ is the coefficient of friction $[\mathrm{kg} / \mathrm{ms}]$ and $d$ is the cross section diameter of the digester $[\mathrm{m}], \Omega=R_{1}\left(\epsilon_{\mathrm{c}}^{2} / \epsilon_{1}\right)+R_{2} \epsilon_{\mathrm{c}}\left|v_{\mathrm{c}}-v_{1}\right|$ is the coefficient of friction or flowing resistance between the two phases where $R_{1}\left[\mathrm{~kg} / \mathrm{m}^{3} \mathrm{~s}\right]$ and $R_{2}\left[\mathrm{~kg} / \mathrm{m}^{4}\right]$ are estimated parameters, and $\eta_{\mathrm{c}}$ and $\eta_{1}[\mathrm{~kg} / \mathrm{ms}]$ are coefficients of vertical turbulent viscous eddy diffusion. The form of the flowing resistance is the Ergun equation which takes into account the force of inertia.

The first term on the right side of Eqn. (58) is the force of inertia. The second term is the internal force of pressure of the chip plug where the chip pressure is given by Eqn. (55). The third term gives the external pressure force from the surrounding free liquor, and the rest of the terms are given in the previous description. Eqn. (59), the momentum balance for the incompressible free liquor, contains equivalent terms as for the chip plug except for the, external pressure term from the chip plug, which for obvious physical reasons does not exist, and the force of friction against the side walls, which is negligible. 
The only unknown variable in these equations is the pressure of the free liquid, $p_{1}$. For this, we do not have an equation of state that relates it to the density of the free liquor because of the assumed incompressibility. This is also expressed in definition (3) which results in Eqn. (46). $p_{1}$ can be estimated from different methods, see for example Patankar (1980), based upon the condition that the pressure has to satisfy the continuity equation. Instead of increasing the complexity of the model by such an estimator, the approach of neglecting the dynamics of Eqn. (59) is made. The resulting stationary elliptic equation is then solved for the pressure gradient:

$$
\frac{\partial p_{1}}{\partial z}=-\rho_{1} v_{1} \frac{\partial v_{1}}{\partial z}+\rho_{1} g-\frac{1}{1-\epsilon_{\mathrm{c}}} \Omega\left(v_{1}-v_{\mathrm{c}}\right)+\eta_{1} \frac{\partial^{2} v_{1}}{\partial z^{2}}
$$

where $\epsilon_{\mathrm{c}}$ is substituted for $\epsilon_{\mathrm{l}}$. Note that beside the gravity, the boundary conditions of the liquid pressure are the driving forces in these equations. The boundary conditions for these equations are defined by the equations in the top, extraction and outlet sections.

In light of the small velocity values which occur in a real vessel (typically $1-4 \mathrm{~mm} / \mathrm{s}$ ), one can query including the dynamics in $v_{\mathrm{c}}$, the force of inertia and the eddy diffusion terms in these equations, since these terms are normally compared to the other forces. The answer lies in the property of stability of the set of equations. Simulation results have shown that these terms have important stabilizing effects. The dynamics also help the whole model converge to a stable stationary point of operation.

3.1.3. The liquid circulations. The circulations (Fig. 1) through the heat exchangers are not included in the model because these do not participate directly in the vertical mass flow. No net extraction or addition from these is assumed. The indirect effect of these can however be included by choosing the dry friction factor $\mu$ considerably larger than normal at the points where the screens are mounted.

3.1.4. The extraction section. In the extraction line we assume a control valve controlled by the control signal $u_{\text {extr }}$ (Fig. 3). The pressure down-flow of the valve $p_{\text {extr }}$ as a boundary condition for Eqn. (60), is assumed to be known. The flow through the

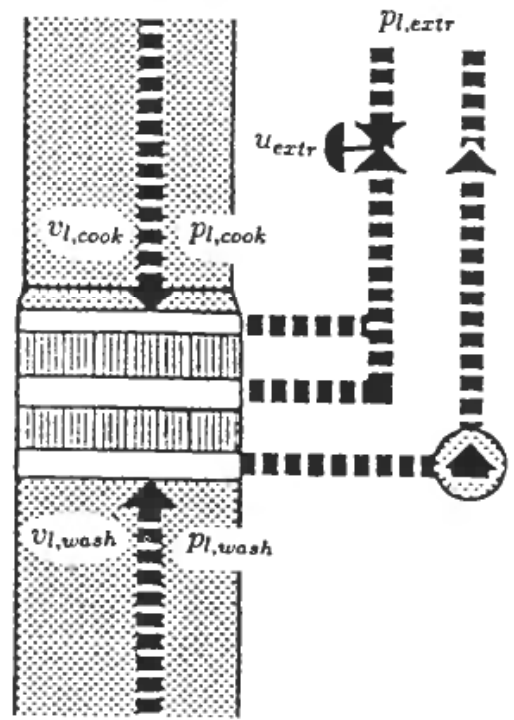

Figure 3. The extraction section. 
valve is a sum of the two flows coming from the cooking zone above with velocity $v_{1 \text {, cook }}$ and from the wash zone below with velocity $v_{1 \text {, wash }}$. Thus, the pressure up-flow of the extraction valve is a superposition of the two pressure contributions resulting from the steam pressure above, $p_{1, \text { cook }}$ and the inlet pressure at the side nozzles in the bottom of the digester, $p_{1 \text {, wash }}$ (Fig 5). These pressures are solved from Eqn. (60).

Given the characteristics of the extraction valve, the two liquid velocities which give the boundary conditions for Eqn. (46), can be written as:

$$
\begin{gathered}
v_{1, \text { cok }}=u_{\text {extr }}\left(p_{1, \text { cook }}-p_{1, \text { extr }}\right)^{1 / 2} /\left(A \epsilon_{\mathrm{c}}\right) \\
V_{1, \text { wash }}=-u_{\text {extr }}\left(p_{1, \text { wash }}-p_{1, \text { extr }}\right)^{1 / 2} /\left(A \epsilon_{\mathrm{c}}\right)
\end{gathered}
$$

where $\epsilon_{\mathrm{c}}$ is the volume fraction of chips in the zone and $A$ is the cross section area of the digester.

\subsection{The top section}

Because it is impossible to determine both density and volume from the mass balance, the volume fraction of chips and free liquid are set fixed in these balance equations, giving the levels as state variables. This error in accuracy is assumed to be of minor importance in light of the small percentage of volume covering this top section compared to the rest of the mass volume.

In the top of the digester, the mass flow from the inlet device is actually decoupled from the flow in the digester. The impulse into the section is therefore a bump against the top of the mass. This tiny effect on the velocity out of the section is neglected. The diffusion and dispersion terms are also neglected. According to Fig. (4), the mass balances of the chip plug and free liquor give rise to the following ordinary differential equations determining the respective levels instead of the volume fractions:

$$
\begin{gathered}
\frac{\partial}{\partial t} h_{\mathrm{c}}=\dot{h}_{\mathrm{c}}=\frac{\rho_{\mathrm{c}, \text { in }} \epsilon_{\mathrm{c}, \text { in }}}{\rho_{\mathrm{c}} \epsilon_{\mathrm{c}}} v_{\mathrm{c}, \text { in }}-v_{\mathrm{c}} \\
\frac{\partial}{\partial t} h_{1}=\dot{h}_{1}=\frac{\rho_{1, \text { in }} \epsilon_{1, \text { in }}}{\rho_{1} \epsilon_{1}} v_{1, \text { in }}-v_{1} .
\end{gathered}
$$

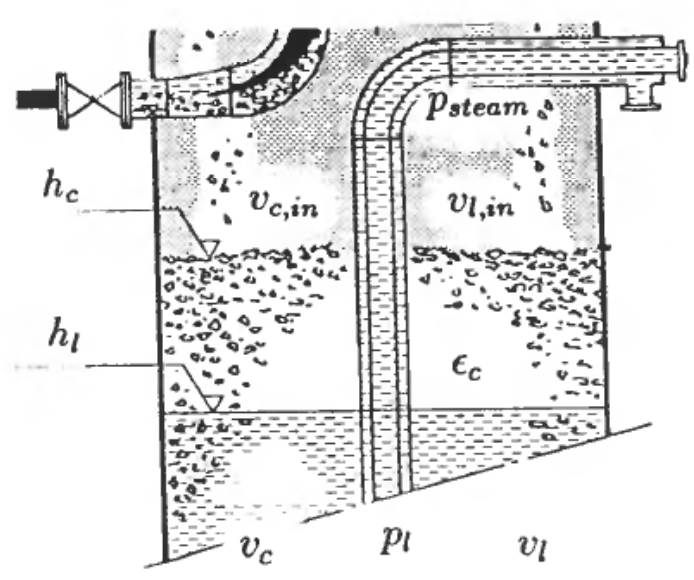

Figure 4. The top section. 
A simple estimation of the volume fraction of chips in the section, assuming a linear relationship to the chip level, can be expressed by:

$$
\epsilon_{\mathrm{c}}= \begin{cases}0.356 & \text { if } h_{\mathrm{c}}>1.0 \mathrm{~m} \\ 0.356+0.022 h_{\mathrm{c}} & \text { otherwise }\end{cases}
$$

The volume fraction in the feed, $\epsilon_{\mathrm{c}, \text { in }}$ is free to vary. The liquid velocity $v_{1}$ is solved from Eqn. (46).

The momentum balance for the free liquid is derived from the same control volume as the mass balances. The steady balance equation gives the liquid pressure at the lower boundary as a function of the steam pressure:

$$
p_{1}=p_{\text {steam }}-\frac{\epsilon_{1, \text { in }} \rho_{1}}{\epsilon_{1}} v_{1, \text { in }} v_{1}+\rho_{1} g h_{1}-\frac{h_{1}}{\epsilon_{1}} \Omega\left(v_{1}-v_{\mathrm{c}}\right) .
$$

Equivalently, the momentum balance equation for the chip plug could be derived based on the same control volume. However, a better estimate for the chip velocity is made by using Eqn. (58).

\subsection{The outlet section}

This section consists of the volume below the bottom scraper covering the inlet of the bottom nozzles. The chip velocity is a function of the rotation speed, $\omega$, of the bottom scraper as long as there is a flow of diluting liquor from the bottom nozzles:

$$
v_{\mathrm{c}, \mathrm{sc}}= \begin{cases}f(\omega) & \text { if } p_{1, \text { wash, bottom }}>p_{1, \text { out }} . \\ 0 & \text { otherwise }\end{cases}
$$

This function has to be established experimentally. $p_{1, \text { wash, bottom }}$ is the liquid pressure given at the inlet of the bottom nozzles, and $p_{\text {out }}$ is the outlet pressure down-flow of the outlet valve which is assumed to be a known boundary condition.

Based on the assumption that the outlet flow is a one-phase flow, which is a good assumption at the low outlet consistency of about $10 \%$, the volume fraction of chips in the outlet can be expressed by:

$$
\dot{\epsilon}_{\mathrm{c}, \text { out }}=\frac{1}{V_{\text {outlet }}}\left(A \epsilon_{\mathrm{c}, \mathrm{sc}} v_{\mathrm{c}, \mathrm{sc}}-\epsilon_{\mathrm{c}, \text { out }} q_{\text {out }}\right)
$$

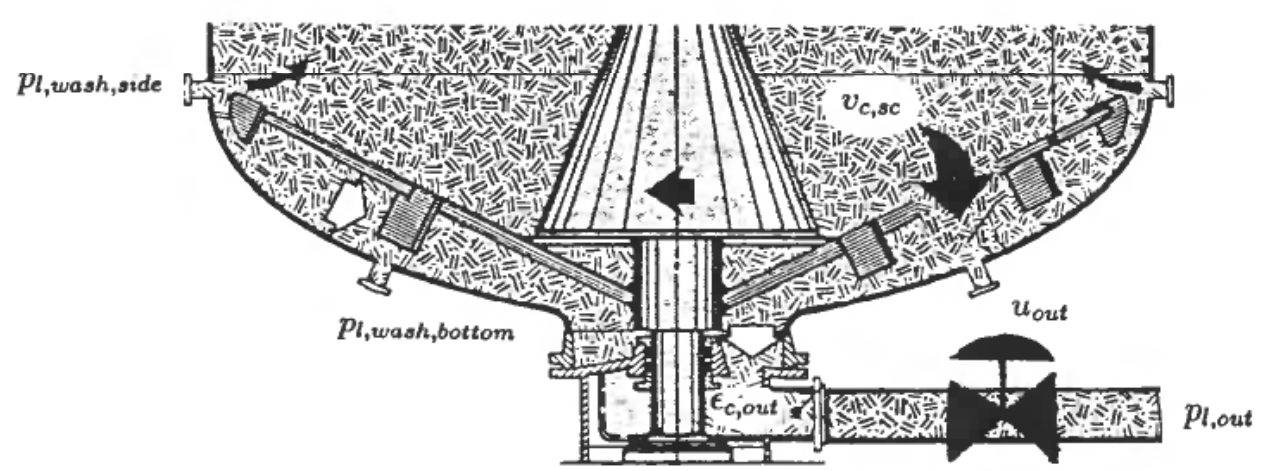

Figure 5. The outlet section. 
where $V_{\text {outlet }}$ is the volume of the section, and equivalently Eqns. (61) and (62):

$$
q_{\text {out }}=u_{\text {out }}\left(p_{1, \text { wash, bottom }}-p_{1, \text { out }}\right)^{1 / 2}
$$

is the total outlet flow of pulp.

\section{Solution of the equations}

Because of the large numerical difference between the values of the volume fraction of chips, density of ions and the chip velocity as state variables in a state space model resulting from discretising the partial differential equations in the space direction, the model becomes unnecessarily stiff. To reduce this artificial stiffness, the model is made dimensionless by scaling the process variables. The characteristic quantities are according to Bird et al. (1960) $d$ the cross-section diameter of the digester, $v_{0}$ typical velocity for both the chip plug and the free liquid, $p_{0}$ a reference pressure, $t_{0}=d / v_{0}$ the characteristic time and $\rho_{0}$ the characteristic densities. The dimensionless variables are $z^{*}=z / d, t^{*}=t / t_{0}, v^{*}=v / v_{0}, p^{*}=\left(p-p_{0}\right) /\left(\rho_{0} v_{0}^{2}\right)$ and $\rho^{*}=\rho / \rho_{0}$. Because the volume fractions and mass fractions are already dimensionless and have values between $0-0$ and $1 \cdot 0$, these are not scaled. The dimensionless partial differential equations are thus:

$$
\begin{aligned}
& \frac{\partial \epsilon_{\mathrm{c}}}{\partial t^{*}}=-\frac{\partial}{\partial z^{*}}\left(\epsilon_{\mathrm{c}} v_{\mathrm{c}}^{*}\right)+\frac{1}{P e_{\mathrm{c}}} \frac{\partial^{2} \epsilon_{\mathrm{c}}}{\partial z^{* 2}} \\
& \frac{\partial v_{1}^{*}}{\partial z^{*}}=\frac{1}{1-\epsilon_{\mathrm{c}}}\left(\left(v_{1}^{*}-v_{\mathrm{c}}^{*}\right) \frac{\partial \epsilon_{\mathrm{c}}}{\partial z^{*}}-\epsilon_{\mathrm{c}} \frac{\partial v_{\mathrm{c}}^{*}}{\partial z^{*}}+\left(\frac{1}{P e_{\mathrm{c}}}-\frac{1}{P e_{1}}\right) \frac{\partial^{2} \epsilon_{\mathrm{c}}}{\partial z^{* 2}}\right) \\
& \frac{\partial \alpha_{\mathrm{lig}}}{\partial t^{*}}=-\frac{\partial}{\partial z^{*}}\left(\alpha_{\mathrm{lig}} v_{\mathrm{c}}^{*}\right)-t_{0} r_{\mathrm{lig}}+\frac{1}{P e_{\mathrm{c}}} \frac{\partial^{2} \alpha_{\mathrm{lig}}}{\partial z^{* 2}} \\
& \frac{\partial \alpha_{\mathrm{carb}}}{\partial t^{*}}=-\frac{\partial}{\partial z^{*}}\left(\alpha_{\mathrm{carb}} v_{\mathrm{c}}^{*}\right)-t_{0} r_{\mathrm{carb}}+\frac{1}{P e_{\mathrm{c}}} \frac{\partial^{2} \alpha_{\mathrm{carb}}}{\partial z^{* 2}} \\
& \frac{\partial \rho_{\text {ion, el }}^{*}}{\partial t^{*}}=\frac{\rho_{\mathrm{ion}, \mathrm{el}}^{*}}{\epsilon_{\mathrm{el}}} \frac{\partial v_{\mathrm{c}}^{*}}{\partial z^{*}}-v_{\mathrm{c}}^{*} \frac{\partial \rho_{\mathrm{ion}, \mathrm{el}}^{*}}{\partial z^{*}}-t_{0} \frac{\rho_{\mathrm{ODW}}}{\epsilon_{\mathrm{el}}}\left(\left(\frac{b_{\text {lig }}}{\rho_{\mathrm{ion}, 0}}+\frac{\rho_{\mathrm{ion}, \mathrm{el}}^{*}}{\rho_{\mathrm{lig}}}\right) r_{\mathrm{lig}}\right. \\
& \left.+\left(\frac{b_{\text {carb }}}{\rho_{\text {ion }, 0}}+\frac{\rho_{\text {ion, el }}^{*}}{\rho_{\text {carb }}}\right) r_{\text {carb }}\right)-\frac{1}{S c_{\text {ion }} \epsilon_{\mathrm{c}} \epsilon_{\mathrm{el}}}\left(\rho_{\text {ion, el }}^{*}-\rho_{\text {ion, } \mathrm{fl}}^{*}\right)+\frac{1}{P e_{\mathrm{c}}} \frac{\partial^{2} \rho_{\text {in, el }}^{*}}{\partial z *^{2}} \\
& \frac{\partial \rho_{\mathrm{ds}, \mathrm{el}}^{*}}{\partial t^{*}}=\frac{\rho_{\mathrm{ds}, \mathrm{el}}^{*}}{\epsilon_{\mathrm{el}}} \frac{\partial v_{\mathrm{c}}^{*}}{\partial z^{*}}-v_{\mathrm{c}}^{*} \frac{\partial \rho_{\mathrm{ds}, \mathrm{el}}^{*}}{\partial z^{*}}-t_{0} \frac{\rho_{\mathrm{ODW}}}{\epsilon_{\mathrm{el}}}\left(\left(\frac{\rho_{\mathrm{ds}, \mathrm{el}}^{*}}{\rho_{\mathrm{lig}}}-\frac{1+b_{\mathrm{lig}}}{\rho_{\mathrm{ds}, 0}}\right) r_{\mathrm{lig}}\right. \\
& \left.+\left(\frac{\rho_{\mathrm{ds}, \mathrm{el}}^{*}}{\rho_{\mathrm{carb}}}-\frac{\left(1+b_{\mathrm{carb}}\right)}{\rho_{\mathrm{ds}, 0}}\right) r_{\mathrm{carb}}\right)-\frac{1}{S c_{\mathrm{ds}} \epsilon_{\mathrm{c}} \epsilon_{\mathrm{el}}}\left(\rho_{\mathrm{ds}, \mathrm{el}}^{*}-\rho_{\mathrm{ds}, \mathrm{fl}}^{*}\right)+\frac{1}{P e_{\mathrm{c}}} \frac{\partial^{2} \rho_{\mathrm{ds}, \mathrm{el}}^{*}}{\partial z^{* 2}} \\
& \frac{\partial \rho_{\mathrm{w}, \mathrm{chip}}}{\partial t^{*}}=-\frac{\partial}{\partial z^{*}}\left(\rho_{\mathrm{w}, \mathrm{chip}} v_{\mathrm{c}}^{*}\right)+\frac{1}{S c_{\mathrm{w} 1} \epsilon_{\mathrm{c}}} \frac{\rho_{\mathrm{ion}, 0}}{\rho_{\mathrm{w}, 0}}\left(\rho_{\text {ion, el }}^{*}-\rho_{\text {ion, fl }}^{*}\right) \\
& +\frac{1}{S c_{\mathrm{w} 2} \epsilon_{\mathrm{c}}} \frac{\rho_{\mathrm{ds}, 0}}{\rho_{\mathrm{w}, 0}}\left(\rho_{\mathrm{ds}, \mathrm{el}}^{*}-\rho_{\mathrm{ds}, \mathrm{f} 1}^{*}\right)+\frac{1}{P e_{\mathrm{c}}} \frac{\partial^{2} \rho_{\mathrm{w}, \mathrm{chip}}^{+}}{\partial z^{* 2}} \\
& \frac{\partial \rho_{\mathrm{in}, \mathrm{fl}}^{*}}{\partial t^{*}}=-\frac{\partial}{\partial z^{*}}\left(\rho_{\mathrm{ion}, \mathrm{fl}}^{*} v_{1}^{*}\right)+\frac{1}{S c_{\mathrm{ivr}} \epsilon_{1}}\left(\rho_{\mathrm{ion}, \mathrm{el}}^{*}-\rho_{\mathrm{in}, \mathrm{fl}}^{*}\right)+\frac{1}{P e_{1}} \frac{\partial^{2} \rho_{\mathrm{ion}, \mathrm{fl}}^{*}}{\partial z^{* 2}}
\end{aligned}
$$




$$
\begin{aligned}
\frac{\partial \rho_{\mathrm{ds}, \mathrm{fl}}^{*}=}{\partial t^{*}}= & -\frac{\partial}{\partial z^{*}}\left(\rho_{\mathrm{ds}, \mathrm{fl}}^{*} v_{1}^{*}\right)+\frac{1}{S c_{\mathrm{ds}} \epsilon_{1}}\left(\rho_{\mathrm{ds}, \mathrm{el}}^{*}-\rho_{\mathrm{ds}, \mathrm{fl}}^{*}\right)+\frac{1}{P e_{1}} \frac{\partial^{2} \rho_{\mathrm{ds}, \mathrm{fl}}^{*}}{\partial z^{* 2}} \\
\frac{\partial \rho_{\mathrm{w}, \mathrm{fl}}^{*}}{\partial t^{*}}= & -\frac{\partial}{\partial z^{*}}\left(\rho_{\mathrm{w}, \mathrm{fl}} v_{1}^{*}\right)-\frac{1}{S c_{\mathrm{w} 1} \epsilon_{1}} \frac{\rho_{\mathrm{ion}, 0}}{\rho_{\mathrm{w}, 0}}\left(\rho_{\mathrm{ion}, \mathrm{el}}^{*}-\rho_{\mathrm{ion}, \mathrm{fl}}^{*}\right) \\
& -\frac{1}{S c_{\mathrm{w} 2} \epsilon_{1}} \frac{\rho_{\mathrm{ds}, 0}}{\rho_{\mathrm{w}, 0}}\left(\rho_{\mathrm{ds}, \mathrm{el}}^{*}-\rho_{\mathrm{ds}, \mathrm{fl}}^{*}\right)+\frac{1}{P e_{1}} \frac{\partial^{2} \rho_{\mathrm{w}, \mathrm{fl}}^{*}}{\partial z^{* 2}} \\
\frac{\partial v_{\mathrm{c}}^{*}}{\partial t^{*}}= & -v_{\mathrm{c}}^{*} \frac{\partial v_{\mathrm{c}}^{*}}{\partial z^{*}}-\frac{\rho_{\mathrm{c}, 0}}{\rho_{\mathrm{c}}^{* \prime} \epsilon_{\mathrm{c}}} \frac{\partial p_{\mathrm{c}}^{*}}{\partial z^{*}}-\frac{\rho_{\mathrm{l}, 0}}{\rho_{\mathrm{c}}^{* \prime}} \frac{\partial p_{1}^{*}}{\partial z^{*}}+\frac{1}{F r}-\frac{d}{v_{0}^{2} \epsilon_{\mathrm{c}} \rho_{\mathrm{c}}^{* \prime}} F_{\mu} \\
& -\frac{\rho_{\mathrm{c}, 0}}{\rho^{* \prime} \mathrm{Re}_{\Omega, \mathrm{c}} \epsilon_{\mathrm{c}}}\left(v_{\mathrm{c}}^{*}-v_{1}^{*}\right)+\frac{\rho_{\mathrm{c}, 0} \partial^{2} v_{\mathrm{c}}^{*}}{\rho_{\mathrm{c}}^{* \prime} R e_{\mathrm{c}} \partial z^{* 2}} \\
\frac{\partial p_{1}^{*}}{\partial z^{*}}= & -\frac{\rho_{1}^{* \prime}}{\rho_{\mathrm{l}, 0}^{*}} v_{1}^{*} \frac{\partial v_{1}^{*}}{\partial z^{*}}+\frac{\rho_{\mathrm{c}}^{* \prime}}{\rho_{\mathrm{l}, 0}} \frac{1}{F r}-\frac{1}{R e_{\Omega, 1}\left(1-\epsilon_{\mathrm{c}}\right)}\left(v_{1}^{*}-v_{\mathrm{c}}^{*}\right) \\
& +\frac{1}{R e_{1}} \frac{\partial^{2} v_{1}^{*}}{\partial z^{* 2}}
\end{aligned}
$$

where the Peclet numbers $P e_{\mathrm{c}}=v_{0} d / D_{\mathrm{c}}$ and $P e_{1}=v_{0} d / D_{1}$ express the relationship between the convective and the dispersive terms for the chip plug and the free liquid respectively, the Schmidt numbers $S c_{\text {ion }}=v_{0} /\left(D_{\text {ion }} d\right)$ and $S c_{\mathrm{ds}}=v_{0} /\left(D_{\mathrm{ds}} d\right)$ express the relationship between the convective mass transport and the molecular diffusion between the free and the entrapped liquid. The Froude number $F r=v_{0}^{2} /(d g)$ expresses the relationship between the force of inertia and the force of gravity, the Reynolds number $R e_{\mathrm{c}}=\rho_{\mathrm{c}, 0} d v_{0} / \eta_{\mathrm{c}}$ expresses the relationship between the force of inertia and the viscous force connected to the isolated flow of the chip plug, $R e_{\Omega, \mathrm{c}}=\rho_{\mathrm{c}, 0} v_{0} /(\Omega d)$ expresses the relationship between the force of inertia and the viscous friction force between the chip plug and the free liquor, and $R e_{1}=\rho_{1,0} d v_{0} / \eta_{1}$ and $R e_{\Omega, 1}=\rho_{1,0} v_{0} /(\Omega d)$ express the equivalent relationships for the free liquor $\rho_{\mathrm{i}}^{*}, i=c, l$ are $\rho_{\mathrm{i}}$ with the respective reference values $\rho_{\mathrm{ion}, 0}, \rho_{\mathrm{ds}, 0}$ and $\rho_{\mathrm{w}, 0}$ inserted. The equations for the top, extraction and bottom sections are scaled equivalently.

These equations are discretized in the space direction $z$ by using the MAC (marker and cell) method by Welch et al.(1966). This staggered grid method where the mass and momentum balances are solved in overlapping control volumes with central differences, is well suited for this model because of the parabolic structure of the partial differential equations, but mostly because of the pressures (or the densities) and velocities dependency of both upstream and downstream conditions, i.e. the gradient terms for both are substantial. The resulting ordinary differential equations are simulated by using MATLAB/SIMULINK.

\begin{tabular}{ll}
\hline Production rate & $22401 \mathrm{chips} / \mathrm{min}$ \\
Steam pressure & $685 \mathrm{kPa}$ \\
Extraction downstream pressure & $750 \mathrm{kPa}$ \\
Side nozzles inlet pressure & $1027 \mathrm{kPa}$ \\
Nominal chip level & $43 \mathrm{~m}$ \\
Nominal liquid level & $41 \mathrm{~m}$ \\
\hline
\end{tabular}

Table 1. Operational conditions for the steam-phase digester model. 


\section{Simulation results}

The operational conditions for a digester model $50 \mathrm{~m}$ high and with a cross section area of $15.9 \mathrm{~m}^{2}$ is listed in Table 1 . A simulation trial has been performed for a vertical discretization of 9 grid points or subsections of the middle section. This forms a state space model of 110 states. The dimensionless parameters for $\epsilon_{\mathrm{c}}=0.6$ are $P e_{\mathrm{c}}$ $=P e_{1}=2 \cdot 7, \quad S c_{\text {ion }}=S c_{\mathrm{ds}}=0 \cdot 4, \quad F r=8 \cdot 2 \cdot 10^{-7}, \quad R e_{\mathrm{c}}=8846 \cdot 1, \quad R e_{\Omega, \mathrm{c}}=8 \cdot 5 \cdot 10^{-5} \quad R e_{1}$ $=8692 \cdot 3$ and $R e_{\Omega, 1}=8 \cdot 4 \cdot 10^{-5}$. Thus, the characteristic flow movement of each phase is turbulent because of the low viscous forces connected to eddy diffusion. The viscous forces between the two phases are, however, dominant.

Figure 6 shows moment profiles for the entrapped and free ions and dissolved solids, and the left hand side of Fig. 7 shows the $\kappa$-number profile. The lignin, carbohydrate and ion concentrations all decrease downwards in the cooking zone such that the $\kappa$ number is reduced from 210 to 60 . Because of this, the amount of dissolved solids increases downwards. The dissolved solids are removed by the extraction. The quenching by the upper wash circulation just below the extraction stops the chemical reactions and therefore the lignin and carbohydrate concentrations are maintained throughout the wash zone. The entrapped ions and dissolved solids are extracted by the counter-current wash flow. The figures show that the concentration of entrapped ions is below the concentration of free ions in the cooking zone while the opposite situation is the case in the wash zone. This is due to the time constant caused by the diffusion coefficient. Also due to this time constant, the figures show that the concentration of
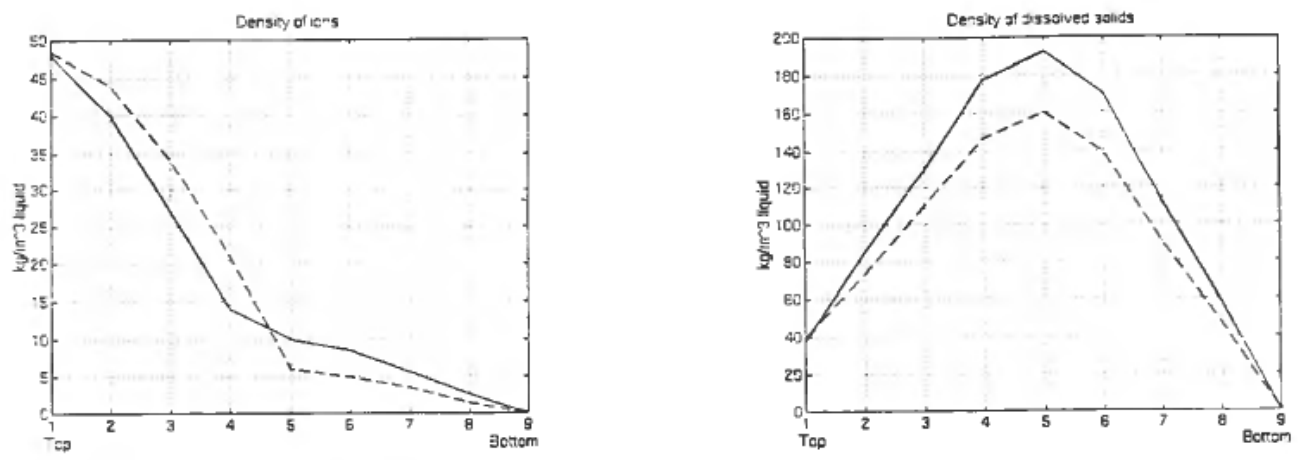

Figure 6. Profiles of the entrapped (solid line) and the free (dashed line) densities of ions and dissolved solids.
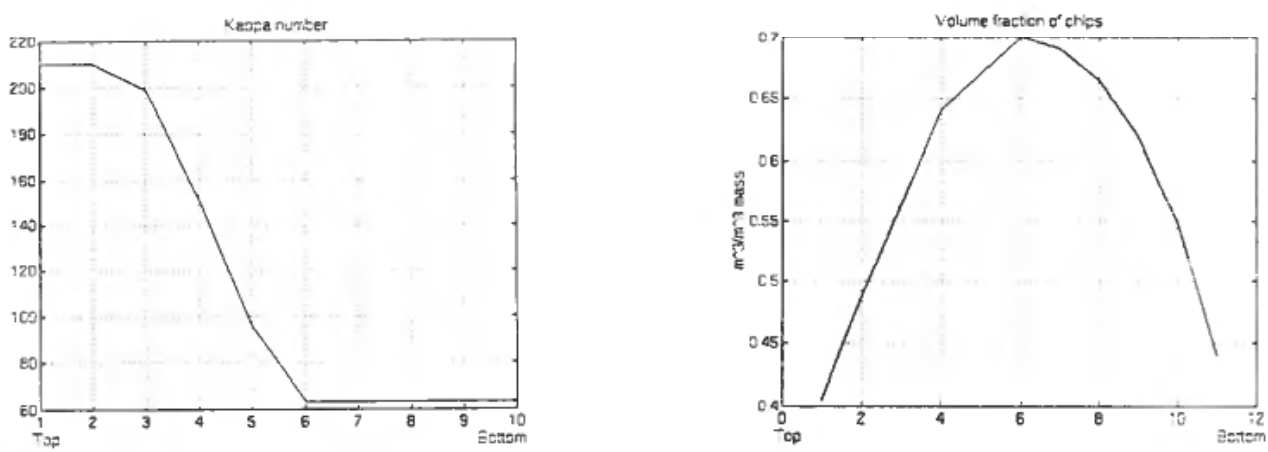

Figure 7. Profiles of $\kappa$ number and the volume fraction of chips. 

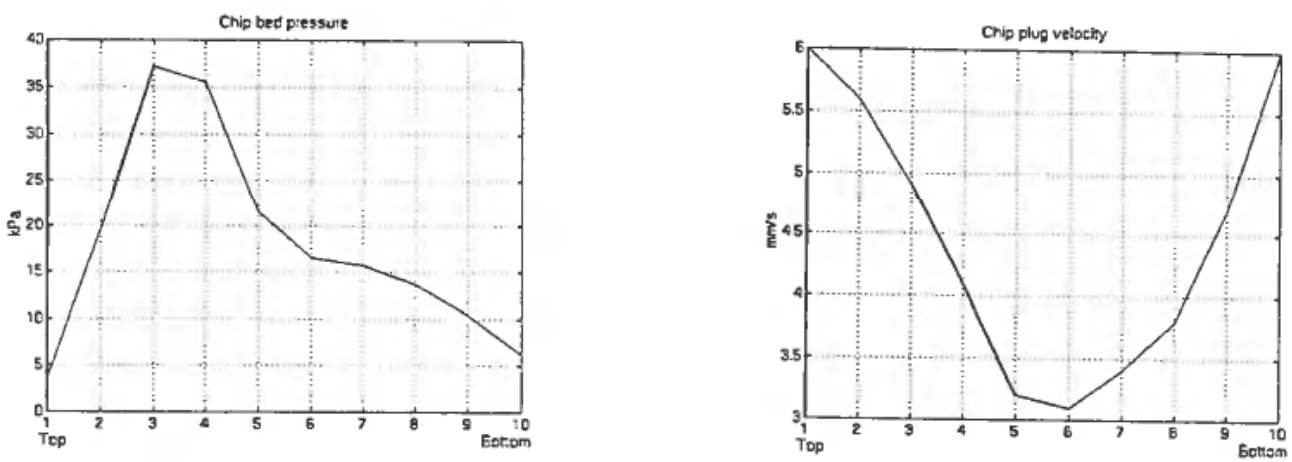

Figure 8. Profiles of the chip pressure and velocity.
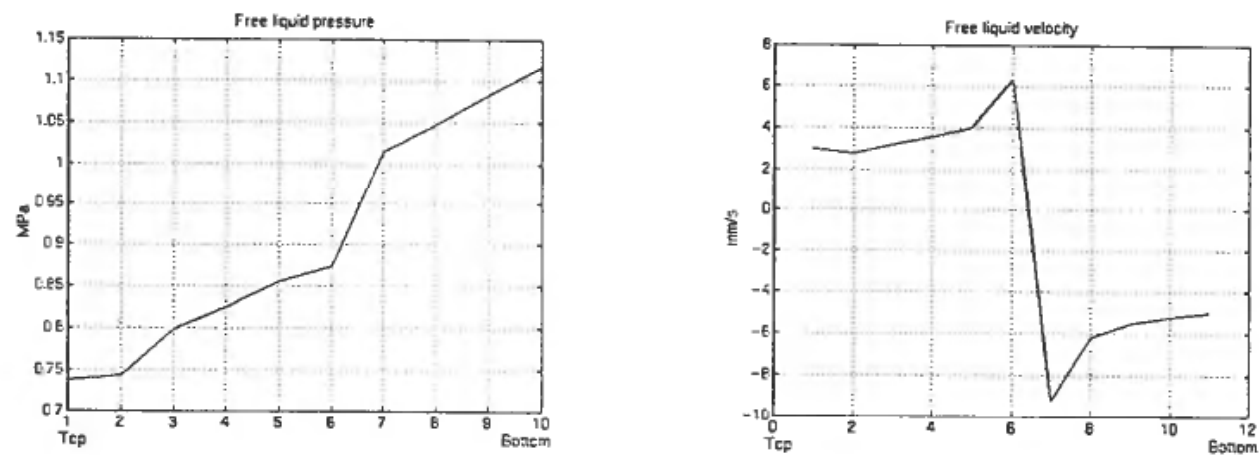

Figure 9. Profiles of the free liquid pressure and velocity.
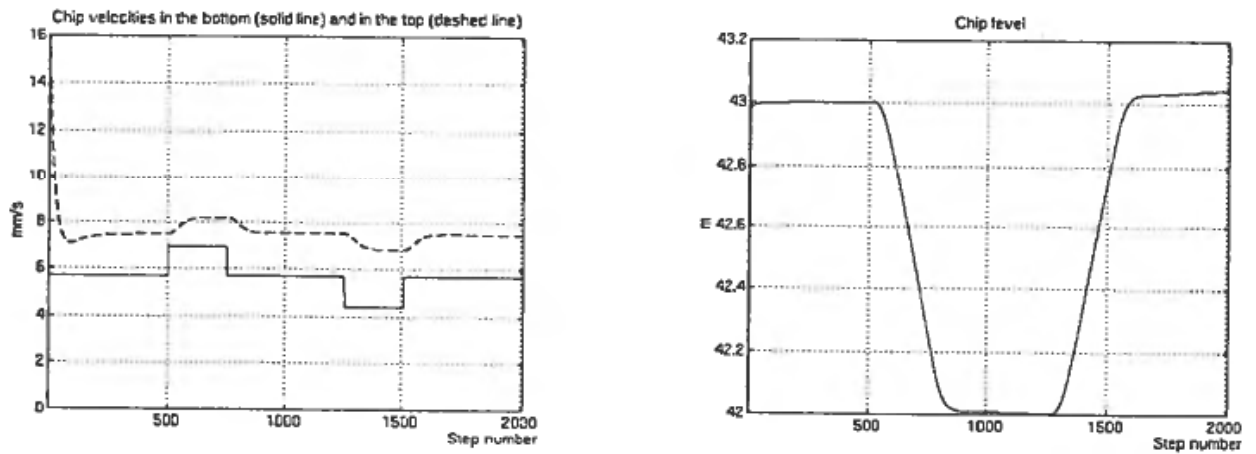

Figure 10. The effects on the chip velocity and the chip level of a pulse pattern in the bottom scraper speed. 
free dissolved solids is below the concentration of entrapped dissolved solids in the vessel as a whole.

Figures 7,8 and 9 show typical profiles for the volume fraction of chips and the pressures and velocities for the chips and the free liquid. The volume fraction of chips increases down towards the extraction. This is due to gravity and the effect is enhanced by the delignification. Thereby, the chip velocity is reduced downwards in the cooking zone. Increasing velocity downwards in the wash zone is due to decreasing compaction as a result of the lift from the upward flowing wash liquid. The chip pressure is related to the compaction and the $\kappa$ number through Eqn. (55).

The vertical liquid velocity increases downwards in the cooking zone. This is both due to the positive gradient of the compaction (increased flowing resistance) and the negative gradient of the chip velocity. It is also driven by the suction pressure from the extraction pump through the screens. Equivalent conditions arise in the wash zone.

The liquid pressure increases from the top towards the extraction because of gravity, but is limited by the suction pressure from the extraction. It is also partly influenced by the difference in the phase velocities. The same condition arises in the wash zone. Note the discontinuity by the extraction in the liquid velocity and pressure.

Figure 10 shows one important dynamical behaviour of the model. This has influence on the chip level control. The bottom scraper excites the model by the pulse pattern shown by the solid line on the left hand figure. The pulse height is about $20 \%$ of the nominal value of the scraper speed. The effect on the chip velocity which excites the chip level $\left(v_{\mathrm{c}}\right.$ in Eqn. (63)) is shown by the dashed line. The right hand figure then shows the effect on the chip level. The simulation time is $1 \mathrm{~h} 20 \mathrm{~min}$.

One of the least certain relationships in this model is obviously the empirical compressibility equation (55), which contributes strongly towards giving the model the form of a pseudo first principles model. A larger span might be expected in the values of volume fraction of chips. The relatively small span, which is shown in Fig. 9, is due to the relatively steep curve of the chip pressure, $p_{\mathrm{c}}$, as a function of volume fraction of chips, $\epsilon_{\mathrm{c}}$. Small variations in $\epsilon_{\mathrm{c}}$ give large variations in $p_{\mathrm{c}}$, which, further, give large variations in the chip velocity, $v_{\mathrm{c}}$.

In all, the model shows promising behaviour, but still has to be tuned for more realistic profiles based on a real digester.

\section{Conclusion}

Modeling the mass flow together with the reaction kinetics for a continuous steam/liquor phase digester is possible in a fairly straightforward manner by considering the mass flow as a two-phase flow and using the conservation laws for mass and momentum to derive partial differential equations. By making certain assumptions, the equations based on the well-known Euler formulation can be solved with boundary conditions given by separate ordinary differential equations and algebraic equations in the top, extraction, and the outlet sections.

By modeling the compaction and the velocity of the chip plug, which are key variables in the process, a valuable tool for controlling the delignification can be achieved. A distributed model like this contains many parameters, the values of which have to be based on different tests, approximations and assumptions. In addition, the many modeled distributed variables are impossible to verify by data from the process measurements most commonly used. Despite this, provided that the parameters are chosen or estimated properly, the resulting model may be more easily tuned against a real 
plant, than would be a simple model. The basic assumption behind this is that the model incorporates a correct structure of the physical mechanisms in the process.

The most important result of this model work is its contribution towards expanding our understanding of the different phenomena arising in these cost consuming industrial processes.

\section{Extensions}

Extensions of this model may follow several paths. First, from a quality analysis point of view it is interesting to model the chip quality, which can be described by variations in the chip moisture and content of lignin and carbohydrates. Also variations in the concentration of active ions, which is expressed by the concentration of caustic soda and the sulphidity etc in the free liquor in the feed, is important. Second, the energy balance may be included to describe the temperature in the digester. If there are substantial temperature variations in the chip feed and/or problems with the heating of the chips by the high pressure steam in the top, a separate energy balance should be developed for the chip plug. The temperature is coupled to the mass balances through the condensing of steam and through the Arrhenius coefficients in the reaction terms. Third, to tune the model against a real plant, the effect of conic side walls may have to be included. Finally, the modeling of a preimpregnation vessel is trivial, based on the same proposed equations.

\section{ACKNOWLEDGEMENTS}

This work was supported by the Research Council of Norway and M. Peterson \& Søn A/S under doctoral scholarship. Special thanks are expressed to Dr. Peder Kleppe and M.Sc. Per Kirkebak of M. Peterson \& Søn A/S, and Dr. Tor Christensen of Fantoft Process A/S.

\section{REFERENCES}

BeAR, J. (1972). Dynamics of Fluids in Porous Media (American Elsevier Publishing Company Inc.).

Bird, R. B., Steward, W. E. and Lightfoot, E. N. (1960). Transport Phenomena (Wiley International Editions).

Christensen, T., Albright, L. F., and Williams, T. J. (1982). A mathematical model of the Kraft pulping process, Report nr 129, Purdue Laboratory for applied industrial control.

FOGLER, H. S. (1992). Elements of chemical reaction engineering. 2nd edition (Prentice-Hall International Editions).

HÄRKonen, E. J. (1984). A mathematical model for two-phase flow, Acta Polytechnica Scandinavica, Mechanical Series 88.

Patankar, S. V. (1980). Numerical Heat Transfer and Fluid Flow (McGraw-Hill Book Company).

Rydholm, S. A. (1967). Pulping Processes (Interscience Publishers, New York).

Sмith, C. C., and Williams, T. J. (1974). Studies of the mathematical modelling, simulation and control of the operation of a Kamyr continuous digester for the Kraft process, Purdue University Engineering, Electronics and Electrical.

Welch, J. E., Harlow, F. H., Shannon, J. P., and Daly, B. J. (1966). The MAC method, Report LA3425, Los Alamos Scientific Laboratory, Los Alamos, N.M. 
Nomenclature

\section{Symbol Description}

$A$ cross section area of the digester

$A_{\mathrm{i}}$ factor of frequency

$b_{\text {lig }}$ stoichiometric coefficient for the reaction with lignin $\mathrm{kg} / \mathrm{kg}$

$b_{\text {carb }}$ stoichiometric coefficient for the reaction with carbohydrates

$D_{\text {ion }}$ mass transfer coefficient for ions in the transfer between the free and entrapped liquid

$D_{\mathrm{ds}}$ mass transfer coefficient for dissolved solids in the transfer between the free and entrapped liquid

$D_{w 1}$ mass transfer coefficient for water in the transfer of ions between the free and entrapped liquid

$D_{\text {w2 }}$ mass transfer coefficient for water in the transfer of dissolved solids between the free and entrapped liquid

$D_{\text {c }}$ dispersion coefficient for the chip plug

$D_{1}$ dispersion coefficient for the free liquid

$E_{\mathrm{i}}$ energy of activation per mol

$f(\omega)$ functional relationship between the rotation speed of the bottom scraper and the chip plug velocity

$F_{\Omega}$ force of viscous interphase friction

$F_{\mu}$ force of dry friction between the chip plug and the side walls

$g$ the constant of gravity

$h_{\mathrm{c}}$ the chip level

$h_{1}$ the free liquid level

$k_{\mathrm{i}}$ Arrhenius coefficients $i=1,2$

$p_{\mathrm{c}}^{\prime}$ chip plug (bed) pressure

$p_{c}$ mean chip plug (bed) pressure

$p_{1}$ free liquid pressure

$p_{1, \text { cook }}$ free liquid pressure ahead of the valve in the extraction line for the calculation of $v_{1, \text { cook }}$

$p_{1, \text { wash }}$ free liquid pressure ahead of the valve in the extraction line for the calculation of $v_{1, \text { wash }}$ side nozzle inlet pressure

$p_{1 \text {, out }}$ bottom nozzle inlet pressure

$p_{\text {steam }}$ downstream pressure for the valve in the blow line

the steam pressure in the top of the digester

$q_{\text {out }}$ the volume flow of pulp out of the outlet section

$r_{\text {lig }}$ lignin mass which is reacting chemically

$r_{\text {carb }}$ carbohydrate mass which is reacting chemically

$r$ the sum of $r_{\text {lig }}$ and $r_{\text {carb }}$

$R_{1}$ coefficient in the viscous friction term

$R_{2}$ coefficient in the viscous friction term

$S$ side wall area of the digester

$t$ time coordinate

$u_{\text {extr }}$ opening variable for the valve in the extraction line

$u_{\text {out }}$ opening variable for the valve in the blow line

$v_{\mathrm{c}}$ chip plug velocity

$v_{c, \text { in }}$ chip velocity in the steam zone

$v_{c, s c}$ chip velocity through the bottom scraper

$v_{c, \gamma}$ interphase chip material velocity

$v_{1}$ free liquor velocity

$v_{1, \text { in }}$ free liquor velocity in the steam zone

$v_{1, \text { cook }}$ free liquor velocity out of the cooking zone

$v_{1 \text {, wash }}$ free liquor velocity out of the washing zone

$V_{\text {out }}$ the volume of the outlet section

$Y$ the quality variable yield

$z$ vertical space coordinate

$\mathrm{kg} / \mathrm{kg}$

$\mathrm{s}^{-1}$

$\mathrm{s}^{-1}$

$s^{-1}$

$\mathrm{s}^{-1}$

$\mathrm{m}^{2} / \mathrm{s}$

$\mathrm{m}^{2} / \mathrm{s}$

$\mathrm{J} / \mathrm{mol}$

$\mathrm{m} / \mathrm{s}$

$\mathrm{N} / \mathrm{m}^{3}$

$\mathrm{N} / \mathrm{m}^{3}$

$\mathrm{m} / \mathrm{s}^{2}$

$\mathrm{m}$

$\mathrm{m}$

(kg ions $/ \mathrm{m}^{3}$ entr. liquids) ${ }^{-1}$

$\mathrm{Pa}$

$\mathrm{Pa}$

$\mathrm{Pa}$

Pa

$\mathrm{Pa}$

$\mathrm{Pa}$

$\mathrm{Pa}$

$\mathrm{Pa}$

$\mathrm{Pa}$

$\mathrm{m}^{3} / \mathrm{s}$

$\mathrm{kg} /(\mathrm{kg}$ ODWs)

$\mathrm{kg} /(\mathrm{kg}$ ODWs)

$\mathrm{kg}$ mass/(kg ODWs)

$\mathrm{kg} /\left(\mathrm{m}^{3} \mathrm{~s}\right)$

$\mathrm{kg} / \mathrm{m}^{4}$

$\mathrm{m}^{2}$

$s$

$\mathrm{m}^{3} /(\mathrm{kg} / \mathrm{m})^{-1 / 2}$

$\mathrm{m}^{3} /(\mathrm{kg} / \mathrm{m})^{-1 / 2}$

$\mathrm{m} / \mathrm{s}$

$\mathrm{m} / \mathrm{s}$

$\mathrm{m} / \mathrm{s}$

$\mathrm{m} / \mathrm{s}$

$\mathrm{m} / \mathrm{s}$

$\mathrm{m} / \mathrm{s}$

$\mathrm{m} / \mathrm{s}$

$\mathrm{m} / \mathrm{s}$

$\mathrm{m}^{3}$

$\mathrm{kg} / \mathrm{kgODW}$

m 
Greek letters

Symbol Description

$\alpha_{\text {lig }}$ mass fraction of lignin in a wood chip

$\alpha_{\text {carb }}$ mass fraction of carbohydrates in a wood chip

$\alpha_{\text {carb }}^{0}$ mass fraction of nonreactive carbohydrates in a wood chip

$\epsilon_{\mathrm{c}} \quad$ volume fraction of wood chips

$\epsilon_{1}$ volume fraction of free liquor

$\epsilon_{\mathrm{i}} \quad$ volume fraction of phase $i$

$\epsilon_{i, \text { in }}$ volume fraction of phase $i$ in the steam zone, $i=c, l$

$\epsilon_{\mathrm{c}, \mathrm{sc}}$ volume fraction of chips by the bottom scraper

$\epsilon_{\mathrm{c} \text {, out }}$ volume fraction of chips in the outlet section

$\epsilon_{\mathrm{el}}$ volume fraction of entrapped liquor in the chips

$\kappa$ the Kappa number

$\Omega$ the viscous friction term

$\eta_{i}$ the coefficient of vertical turbulent viscous eddy diffusion, $i=c, l$

$\rho_{\mathrm{c}}$ mass density of one single wood chip

$\rho_{1}$ mass density of free liquor

$\rho_{c, \text { in }}$ mass density of wood chips in the steam zone

$\rho_{1, \text { in }}$ mass density of free liquor in the steam zone

$\rho_{\mathrm{sm}}$ mass density of the solid materials in the wood chips

$\rho_{\mathrm{el}}$ mass density of the entrapped liquid in the wood chips

$\rho_{\text {ion, el }}$ mass density of the entrapped ions

$\rho_{\mathrm{ds}, \text { el }}$ mass density of the entrapped dissolved solids

$\rho_{\mathrm{w}, \text { chip }}$ mass density of the water in the wood chips

$\rho_{\text {ion,fl }}$ mass density of the free ions

$\rho_{\mathrm{ds}, \mathrm{fl}}$ mass density of the free dissolved solids

$\rho_{\mathrm{w}, \mathrm{fl}}$ mass density of the water in the free liquid

$\rho_{\text {oDw }}$ mass density of one single oven dry chip

Dimension

$\mathrm{kg}$ lignin/kg ODW

kg carb./kg ODW

kg carb./kgODW

$\mathrm{m}^{3}$ chips $/ \mathrm{m}^{3}$ mass

$\mathrm{m}^{3}$ liquor $/ \mathrm{m}^{3}$ mass

$\mathrm{m}^{3} / \mathrm{m}^{3}$ mass

$\mathrm{m}^{3} / \mathrm{m}^{3}$ mass

$\mathrm{m}^{3} / \mathrm{m}^{3}$ mass

$\mathrm{m}^{3} / \mathrm{m}^{3}$ mass

$\mathrm{m}^{3}$ liquor $/ \mathrm{m}^{3}$ ODW

$\mathrm{kg} /\left(\mathrm{m}^{3} \mathrm{~s}\right)$

$\mathrm{kg} /(\mathrm{ms})$

$\mathrm{kg} / \mathrm{m}^{3}$ ODW

$\mathrm{kg} / \mathrm{m}^{3}$ free liquid

$\mathrm{kg} / \mathrm{m}^{3}$ ODW

$\mathrm{kg} / \mathrm{m}^{3}$ free liquid

$\mathrm{kg} / \mathrm{m}^{3}$ ODW

$\mathrm{kg} / \mathrm{m}^{3}$ ODW

$\mathrm{kg} / \mathrm{m}^{3}$ entrapped liquid

$\mathrm{kg} / \mathrm{m}^{3}$ entrapped liquid

$\mathrm{kg} / \mathrm{m}^{3}$ ODW

$\mathrm{kg} / \mathrm{m}^{3}$ free liquid

$\mathrm{kg} / \mathrm{m}^{3}$ free liquid

$\mathrm{kg} / \mathrm{m}^{3}$ free liquid

$\mathrm{kg} / \mathrm{m}^{3}$ ODW

Characteristic numbers

\section{Symbol Description}

d cross diameter used as a reference length

$p_{0}$ reference pressure

$\rho_{0}$ reference density

$t_{0}=d / v_{0}$ characteristic time variable

$v_{0}$ reference velocity

Dimension
$\mathrm{m}$
$\mathrm{Pa}$
$\mathrm{kg} / \mathrm{m}^{3}$
$\mathrm{~s}$
$\mathrm{~m} / \mathrm{s}$

Dimension

$\mathrm{Pa}$

$\mathrm{m} / \mathrm{s}$

\footnotetext{
Dimensionless numbers

Symbol Description

Fr Froude number

Pe Peclet number

Re Reynolds number

Sc Schmidt number
} 